TITLE:

Classification of Polar-Night Jet Oscillations and Their Relationship to Fast and Slow Variations in a Global Mechanistic Circulation Model of the Stratosphere and Troposphere

$\operatorname{AUTHOR}(S):$

Kohma, Masashi; Nishizawa, Seiya; Yoden, Shigeo

\title{
CITATION:
}

Kohma, Masashi .... [et al]. Classification of Polar-Night Jet Oscillations and Their

Relationship to Fast and Slow Variations in a Global Mechanistic Circulation Model of the Stratosphere and Troposphere. Journal of Climate 2010, 23(23): 6438-6444

ISSUE DATE:

2010-12

URL:

http://hdl.handle.net/2433/197170

RIGHT:

(c) Copyright 2010 American Meteorological Society (AMS). 


\title{
NOTES AND CORRESPONDENCE
}

\section{Classification of Polar-Night Jet Oscillations and Their Relationship to Fast and Slow Variations in a Global Mechanistic Circulation Model of the Stratosphere and Troposphere}

\author{
MASASHI KOHMA* \\ Department of Geophysics, Kyoto University, Kyoto, Japan \\ SEIYA NISHIZAWA \\ Department of Earth and Planetary Sciences, Kobe University, Hyogo, Japan \\ SHIGEO YODEN \\ Division of Earth and Planetary Sciences, Graduate School of Science, Kyoto University, Kyoto, Japan
}

(Manuscript received 1 October 2009, in final form 13 June 2010)

\begin{abstract}
Polar-night jet oscillation (PJO), which is a low-frequency intraseasonal oscillatory variation in the winter stratosphere, is analyzed statistically with a 14000 -yr-long dataset obtained with an idealized global mechanistic circulation model of the stratosphere and troposphere. After performing an empirical orthogonal function (EOF) analysis on the low-pass-filtered time series of the northern polar temperature, 10647 PJO events are identified and classified into four groups. About $80 \%$ of them are two groups of warm events while the rest are two groups of cold events, which are newly identified variations with opposite sign from the warm events by the same EOF analysis. All of them show slow downward propagations of a positive or negative temperature anomaly, with a relatively short or long lifetime.

Composite analysis with such a large number of samples shows that each group has its own typical relationship to unfiltered relatively fast variations in the polar stratosphere known as stratospheric sudden warming and polar vortex intensification and to the slow variation in the troposphere known as the Arctic Oscillation. Statistically significant evidence of the downward dynamical influence of PJO on the surface is obtained for a group of warm events with a longer lifetime.
\end{abstract}

\section{Introduction}

During the Northern Hemisphere winter, intraseasonal variations in the stratosphere-troposphere coupled system contain events with a range of characteristic time scales from relatively fast stratospheric sudden warming (SSW) and polar vortex intensification (VI) to slow northern annular mode (NAM) and polar-night jet oscillation (PJO). SSW is a spectacular natural phenomenon associated with a rapid breakdown of the polar vortex because of wave-

\footnotetext{
* Current affiliation: Dept. of Earth and Planetary Science, Graduate School of Science, University of Tokyo, Tokyo, Japan.
}

Corresponding author address: Shigeo Yoden, Division of Earth and Planetary Sciences, Graduate School of Science, Kyoto University, Kyoto, 606-8502, Japan.

E-mail: yoden@kugi.kyoto-u.ac.jp mean flow interactions on planetary scales (e.g., McIntyre 1982), and it is followed by a rather gradual recovery of the polar vortex except for the final warming during spring. On the other hand, VI events have the opposite sign of anomaly variations in zonal-mean temperature and zonal wind to SSW events (Limpasuvan et al. 2005). A VI event is marked by gradually descending cold and westerly anomalies with the absence of a strong planetary wave forcing in the polar stratosphere followed by a relatively quicker recovery after the maximum phase. Asymmetry of the time variations of SSW and VI events is characterized by the difference in the acceleration process of the stratospheric westerly wind: deceleration due to wave driving can be rapid, while acceleration associated with radiative cooling is generally more gradual.

As for the slow variations in the Northern Hemisphere troposphere, the Arctic Oscillation (AO) is one of the well-known slow variations. The term was coined 
by Thompson and Wallace (1998) as the leading pattern of low-frequency variations of the wintertime sea level pressure. They interpreted it as the surface signature of modulations in the strength of the polar vortex aloft, which are now known as NAM variations. Baldwin and Dunkerton (1999) showed the downward propagation of the signals of NAM from the stratosphere to the troposphere with a time lag of about three weeks. Kuroda and Kodera (1999) also showed poleward and downward propagation of zonal-mean zonal wind anomaly in the stratosphere associated with planetary wave variations. Kuroda and Kodera (2001) coined the term PJO for these slow month-to-month variations of the polar-night jet. NAM and PJO are not totally distinct phenomena, but they share to some (possibly large) degree characteristics of slow variations in the polar vortex. In this study, we distinguish the two terms simply by their analysis procedure.

The relationship between these fast and slow variations of the winter polar vortex has been studied with global reanalysis datasets. Kodera et al. (2000) showed an inphase relationship between SSW and PJO and argued a possible "conditioning" for SSW by the slowly varying zonal-mean state. Recently, Kuroda (2008) noted PJOs are slow variations including SSW and VI in their time evolutions and showed an AO pattern is created before and after the occurrence of SSW or VI with opposite polarity. On the slow time scales, Kuroda and Kodera (2004) found that $\mathrm{AO}$ tends to have a longer lifetime when PJO has a sufficient amplitude and preferable phase.

In this study, we investigate the relationship of PJO to the fast variations of SSW and VI, as well as to the slow variation of the surface AO, by using a 14 000-yr dataset obtained from an idealized global mechanistic circulation model of the stratosphere and troposphere. Our numerical experiment produces much larger samples than the reanalysis datasets so that we can obtain results with enough statistical significance, though they are computationally obtained virtual data. In addition, the numerical experiments have merit that internal intraseasonal variations can be isolated from any atmospheric responses to external forcings with similar time scales by prescribing a purely periodic annual forcing.

\section{Data}

The dataset was originally obtained by Nishizawa and Yoden (2005) with an idealized global circulation model under a purely periodic annual radiative forcing. In their model, several simplifications were made, such as Newtonian heating and cooling with prescribed radiative equilibrium temperature, a dry atmosphere without moist processes, Rayleigh friction at the bottom boundary and the top sponge layer, and a sinusoidal surface topography of zonal wavenumber 1 only in the Northern Hemisphere. It should be noted that due to these simplifications it might be difficult to compare our results with the real atmosphere directly. However, the present mechanistic circulation model retains the dynamical processes essential to what we investigate, such as SSW, VI, NAM, and $\mathrm{PJO}$ in the stratosphere and $\mathrm{AO}$ in the troposphere, and due to the simplifications it can be integrated for long enough to obtain statistical significant results.

We analyze polar temperature, $T_{\mathrm{po}}(p, t)$, averaged over latitudes $\phi \geq 60^{\circ} \mathrm{N}$, with a pressure range of $925 \geq p \geq$ $0.1 \mathrm{hPa}$ and time $0 \leq t \leq 360$ days $\times 14000 \mathrm{yr}$. Daily data is used for the analyses of fast SSW and VI events, while their low-passed (15-day running mean) time series $\overline{T_{\text {po }}(p, t)}$ is used for the analysis of slow PJO. This time averaging removes the relatively fast nature of SSWs, whereas slow variations associated with them are retained. We also use the same low-passed time series of zonal-mean surface pressure $\overline{\left[p_{s}\right](\phi, t)}$ for the analysis of slow AO in the troposphere.

\section{Results}

\section{a. Classification of polar-night jet oscillations}

To identify PJO events as Kuroda and Kodera (2004) did, we perform an empirical orthogonal function (EOF) analysis for the time series of $\overline{T_{\text {po }}(p, t)}$, after subtracting the 14 000-yr-mean seasonal march. Figure 1a shows the vertical profiles of two leading EOFs: EOF1 has a large amplitude in the midstratosphere, whereas EOF2 has opposite sign between the upper and the lower stratosphere with a node at $p \sim 10 \mathrm{hPa}$. These two modes account for $75.2 \%$ and $20.1 \%$, respectively, of the total variance. We introduce a two-dimensional phase space spanned by the normalized time coefficients of these components, which was named "PJO space" by Kuroda and Kodera (2004), to investigate slow oscillatory variations in the polar region.

Figure 1b shows trajectories in the PJO space for two typical years. Both trajectories rotate anticlockwise with large amplitudes during winter months, indicating downward propagation of temperature anomalies as deduced from the vertical profiles of the EOFs. The red trajectory has the largest amplitude in the third quadrant, where a warm anomaly is located in the lower stratosphere. On the other hand, the blue trajectory has large amplitudes in the first quadrant, corresponding to a cold anomaly in the lower stratosphere. These trajectories show a variety of slow oscillatory variations in the winter polar region, and we realize the necessity of classifying these variations.

We define a PJO event as a period during which the amplitude in the PJO space is continuously larger than 
(a)

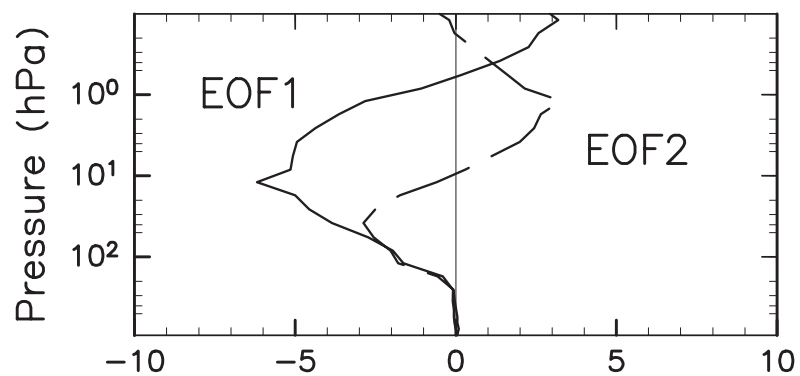

(b)

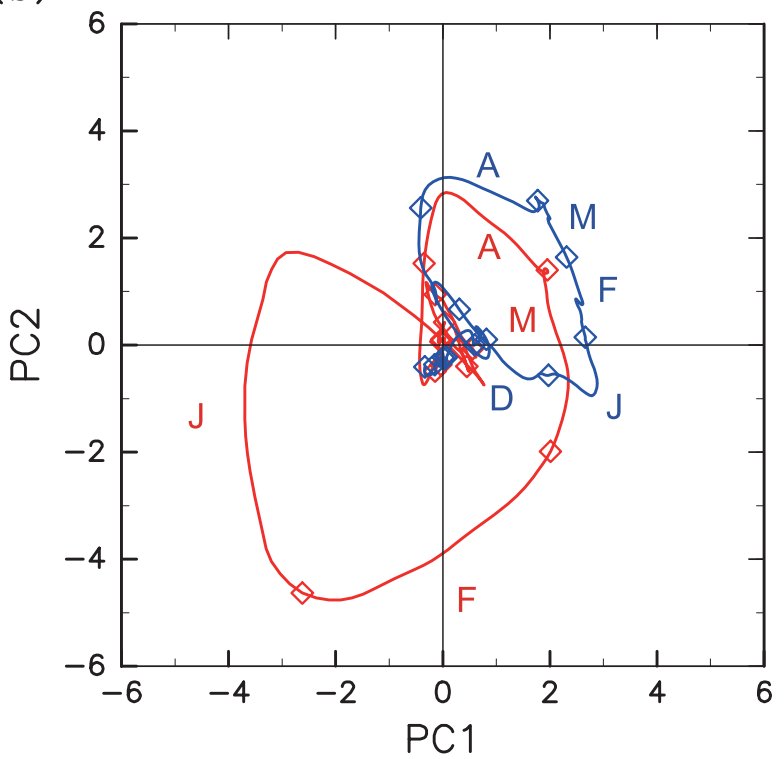

FIG. 1. (a) Vertical profiles of two leading EOFs obtained for the northern polar temperature from 925 to $0.1 \mathrm{hPa}$. (b) Two examples of annual trajectories, starting on 1 Jun, in the two-dimensional PJO space spanned by normalized time coefficients (PC1 and PC2) of the two leading EOFs. Red (blue) line corresponds to a warm (cold) PJO event. Diamonds indicate the first day for each month. December, January, February, March, and April are denoted by their first letter.

two and its maximum exceeds three; Fig. 2a shows a scatter chart of the points of maxima for 10647 events that satisfy this criterion in $8845 \mathrm{yr}$. Note that no PJO event is observed in about one-third of the $14000 \mathrm{yr}$. To classify these PJO events, we use the phase of the state vector for each maximum. As shown by different colors in Fig. 2b, the PJO events are rather well separated into four groups in the histogram of the argument: we call them Warm1 (orange), Warm2 (red), Cold1 (green), and Cold2 (blue) events, based on the temperature anomaly at each maximum. The numbers of these events are 4970, 3488,820 , and 1369 , respectively. Nearly $80 \%$ of the PJO events are warm events, while two groups of cold events are newly identified in this study with the same EOFs but opposite sign from the warm events.
A composite analysis is performed for the four groups about the key day of the maximum amplitude in the PJO space. Panels in the third row of Figs. 3a-d show pressure-time sections of the composite temperature anomaly for 100 days before and after the key day. During the Warm 1 event (Fig. 3a), a positive anomaly over $10 \mathrm{~K}$ appears at $1 \mathrm{hPa}$ at $\mathrm{Lag} \sim-10$ day and propagates downward to $80 \mathrm{hPa}$ within 20 days. The large positive anomaly, which is over $35 \mathrm{~K}$ at the maximum phase in the upper stratosphere, decreases gradually at each level. The decrease is slower in the lower stratosphere, where the anomaly becomes less than $5 \mathrm{~K}$ by Lag $\sim 40$ day. The time evolution of Warm1 is similar to that of SSWs, which occur "suddenly" to attain the maxima, and the warm anomalies last for several weeks longer than the radiative time scale in the stratosphere. During the Warm2 event (Fig. 3b), on the other hand, a positive anomaly over $10 \mathrm{~K}$ appears at $1 \mathrm{hPa}$ at Lag $\sim-40$ day, propagates downward slowly, and reaches $100 \mathrm{hPa}$ on the key day. A large positive anomaly over $30 \mathrm{~K}$ appears in the lower stratosphere. Note that a negative anomaly over $10 \mathrm{~K}$ appears in the mesosphere about 20 days before the key day and propagates downward slowly. The cold anomaly below $-5 \mathrm{~K}$ reaches the midstratosphere around $\mathrm{Lag} \sim 50$ day. The composite lifetime of the Warm2 event is about 60 days and is comparable to a half cycle of observed PJOs (Kodera et al. 2000).

Both Cold 1 and Cold 2 events show slow downward propagation of negative anomalies. During Cold1 events, the composite minimum below $-20 \mathrm{~K}$ appears in the upper stratosphere for $-10 \leq \mathrm{Lag} \leq 10$ day, and it propagates downward while the magnitude decreases slowly. During Cold2 events, on the other hand, a negative anomaly below $-10 \mathrm{~K}$ persists for a longer period of $-80<\mathrm{Lag}<-10$ day at $1 \mathrm{hPa}$, and it propagates downward with gradually increasing magnitude. A negative anomaly below $-20 \mathrm{~K}$ appears in the midstratosphere for a period of $-35 \leq \mathrm{Lag} \leq-5$ day. Note that the maximum of positive anomaly over $25 \mathrm{~K}$ at $1 \mathrm{hPa}$ is around the key day during the Cold 2 event, and the downward propagation of the warm anomaly from the mesosphere to the upper stratosphere is before the key day. Case-to-case variations (i.e., the standard deviation) of the temperature anomaly in the stratosphere are smaller during cold events than during warm events. Cold 1 and Cold 2 events resemble each other except for the phase of each key day, though there are two wellseparated peaks around $-30^{\circ}$ and $90^{\circ}$ in Fig. $2 \mathrm{~b}$.

\section{b. Timing of unfiltered fast variations in each group of $P J O$}

By introducing several threshold values to the North Pole temperature at $2.6 \mathrm{hPa}$, we define two types of 
(a)

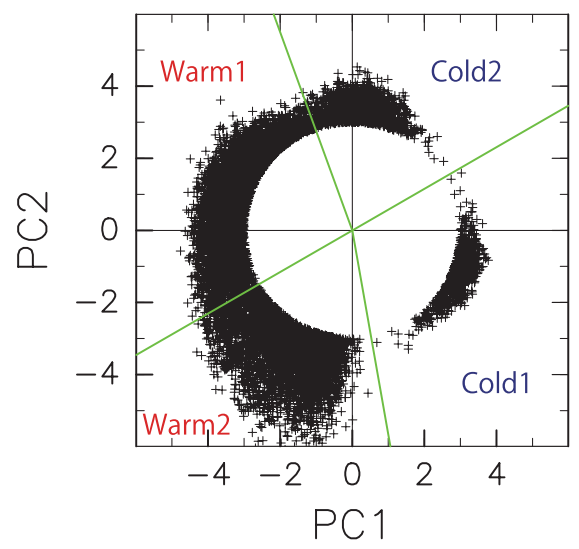

(b)

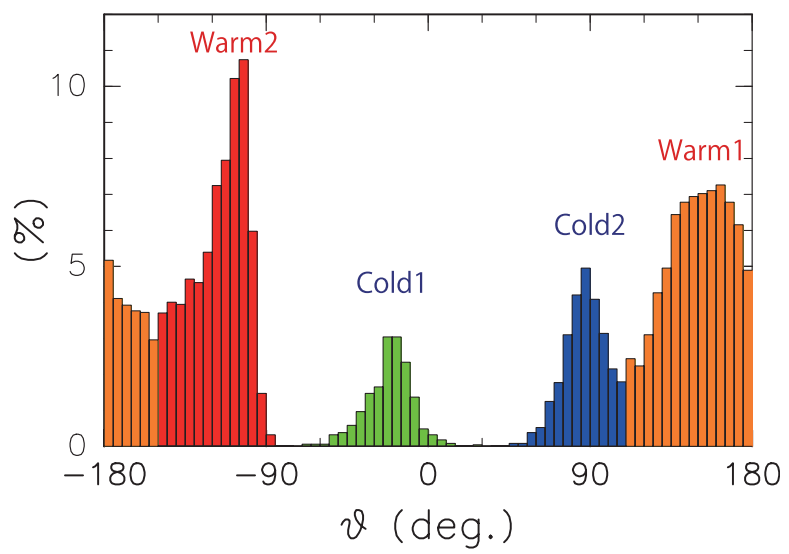

FIG. 2. (a) Scatterchart in the PJO space of the state vector at local amplitude maxima for each PJO event. Four lines indicate $30^{\circ}, 110^{\circ}, 210^{\circ}$, and $280^{\circ}$ from the positive $\mathrm{PC} 1$ axis, which are used in classifying PJOs. (b) Histogram of the phase of the state vector for each maximum in the PJO space.

unfiltered fast variations in the stratosphere: SSW and VI. An SSW event is defined here as a period during which temperature excess from the minimum value in $14000 \mathrm{yr}$ for each calendar day is continuously above $30 \mathrm{~K}$ and its maximum is higher than $60 \mathrm{~K}$. The date of the maximum is the key day of each SSW. In total, 15734 SSW events are identified in $11287 \mathrm{yr}$. A VI event, on the other hand, is defined as a period of 10 days or longer during which a temperature anomaly from the mean value of $14000 \mathrm{yr}$ for each calendar day is continuously below $-5 \mathrm{~K}$, and its minimum at the key day is lower than $-15 \mathrm{~K} ; 13725 \mathrm{VI}$ events are identified in $11091 \mathrm{yr}$. Here, SSW was defined with respect to the minimum temperature directly to see the temperature rise from the minimum state by dynamical process during each SSW event, while VI was defined with the mean temperature to extract a period with relatively weak planetary wave forcing compared to the mean state.

The top panels in Figs. 3a-d show histograms of the key day difference between the first SSW during each winter and each of the four types of PJO events. About $95 \%$ of both Warm 1 and Warm 2 events are accompanied by the SSW events. The timing of the SSW occurrence concentrates around the key day of PJO during Warm 1 events, while most of the SSW events precede the key day of Warm 2 events by 10 days or more with an average lead time of about 25 days. Note the concentration of the timing of SSW occurrence just after the key day of Cold2 events, which corresponds to the maximum of positive temperature anomaly over $25 \mathrm{~K}$ at $1 \mathrm{hPa}$ around the key day as stated in the previous subsection.

The second-row panels in Figs. 3a-d show similar histograms for the key day difference between VI and PJO. Over $99 \%$ of both cold events are accompanied by the VI events. More than half of VI events concentrate around the key day of Cold1 events, while most of VI events precede the key day of Cold 2 events by 15 days or more, with an average lead time of about 40 days. The histograms of VI for both Warm1 and Warm2 events show broad distributions without a sharp peak before or after the key day.

\section{c. Slow tropospheric variations in each group of PJO}

We introduce an AO index that characterizes slow variations of the polar vortex in the troposphere. The leading EOF of a low-passed (15-day running mean) time series of zonal-mean surface pressure with a latitudinal weight $\overline{\left[p_{s}\right](\phi, t)} \cos \phi$ (for $\phi \geq 20^{\circ} \mathrm{N}$ ) explains $77.4 \%$ of the total variance and has a seesaw pattern between the polar region and midlatitudes with a node around $58^{\circ} \mathrm{N}$. The time coefficient of the leading EOF is used as the AO index. It varies all year-round with little seasonal dependence. The autocorrelation of the AO index decreases with a factor of $e$ for about 30 days and becomes nearly zero after about 60 days.

The bottom panels in Figs. 3a-d show the time variations of the composite mean and the standard deviation of the AO index for each PJO group. Both Warm1 and Warm2 events are associated with a positive peak of the AO index, which corresponds to the negative anomaly of surface pressure over the polar region-about 30 and 40 days prior to the key day of PJO, respectively. Both Cold1 and Cold 2 events, on the other hand, are associated with a negative peak of the $\mathrm{AO}$ index about 30 and 45 days prior to the key day, respectively. The maximum absolute values are larger than 1 , which corresponds to the surface pressure deviation of $9 \mathrm{hPa}$ at $80^{\circ} \mathrm{N}$. All of these 
(a) Warm 1
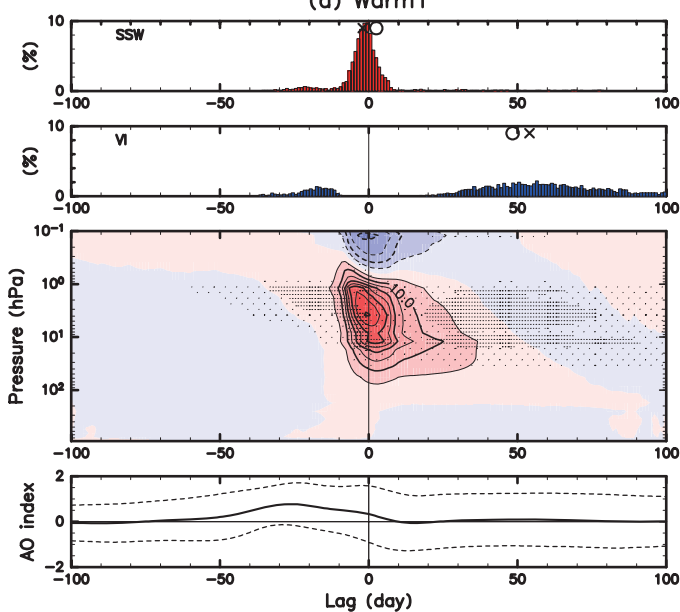

(c) Cold1
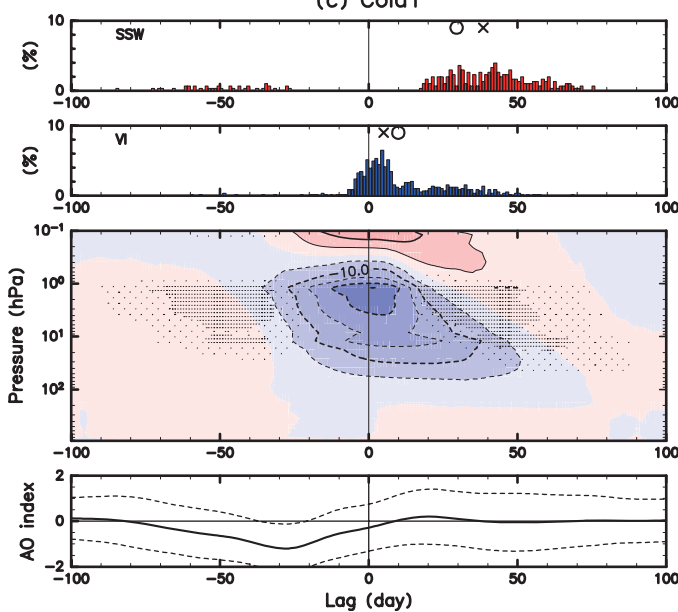
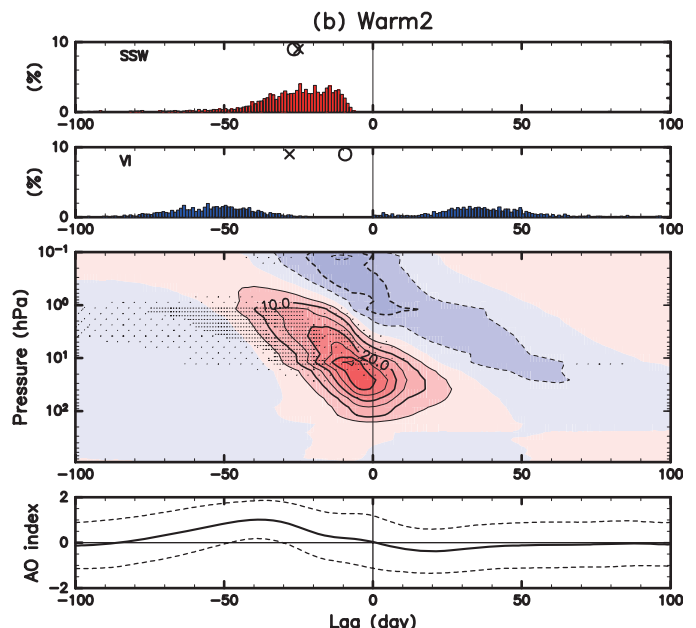

(d) Cold2
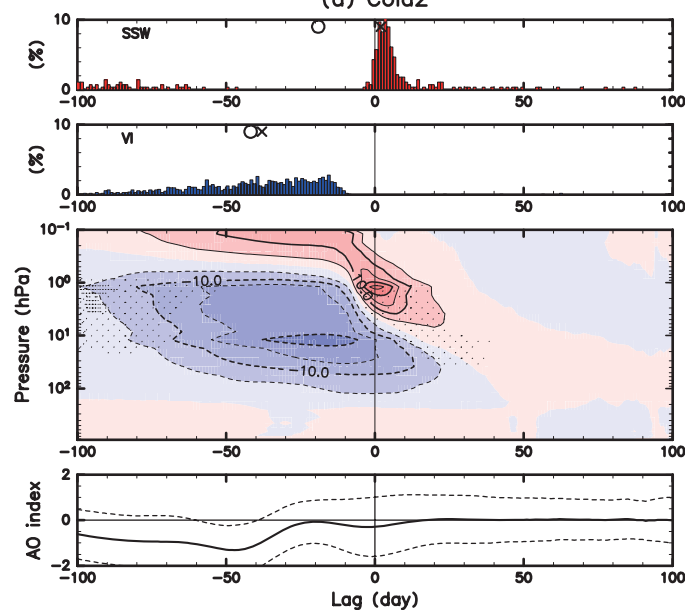

FIG. 3. Composite analyses for (a) Warm1, (b) Warm2, (c) Cold1 and (d) Cold2 PJO events. (top) Histogram of the key day difference between the first SSW during each winter and each PJO event. The circles and crosses indicate mean and median, respectively. (top middle) As in the top row, but for VI. (bottom middle) Pressure-time section of the composite polar temperature anomaly for 100 days before and after the key day of the maximum amplitude in the PJO space. Warm (cold) color is for positive (negative) anomaly. Heavy (light) shade indicates standard deviation greater than 15 (10) K. (bottom) AO index (thick solid line) and the mean AO index plus and minus one standard deviation (dashed lines).

peaks are highly significant because of the large number of samples.

After the key day of each PJO event, the AO index approaches zero quickly except in the case of Warm2 events, for which a comparatively small negative peak (about -0.4 ) appears at Lag $\sim 20$ day and then approaches zero. The confidence level of the deviation of the peak from zero is more than $99.9 \%$ because of the large number of samples (3488 events).

\section{Discussion}

To confirm the oscillatory nature of these four groups of PJOs, trajectories in the two-dimensional PJO space are computed for the composite variations. Composite trajectories for both types of warm events rotate anticlockwise, amplifying in the second quadrant, as shown in Fig. 4a. During Warm1 events, the amplitude decreases comparatively rapidly after attaining its maximum with a lifetime of about 40 days. During Warm 2 events, on the other hand, amplification continues over 40 days with a larger variation of the phase compared to Warm1 events; the composite trajectory has a maximum in the third quadrant, followed by gradual damping with further rotations over about 40 days. Figure $4 \mathrm{~b}$ shows the composite trajectories for Cold 1 and Cold 2 events. These trajectories are not simply the opposite sign of the trajectories for Warm 1 and Warm 2 events, indicating the 
(a) Warm1 \& Warm2

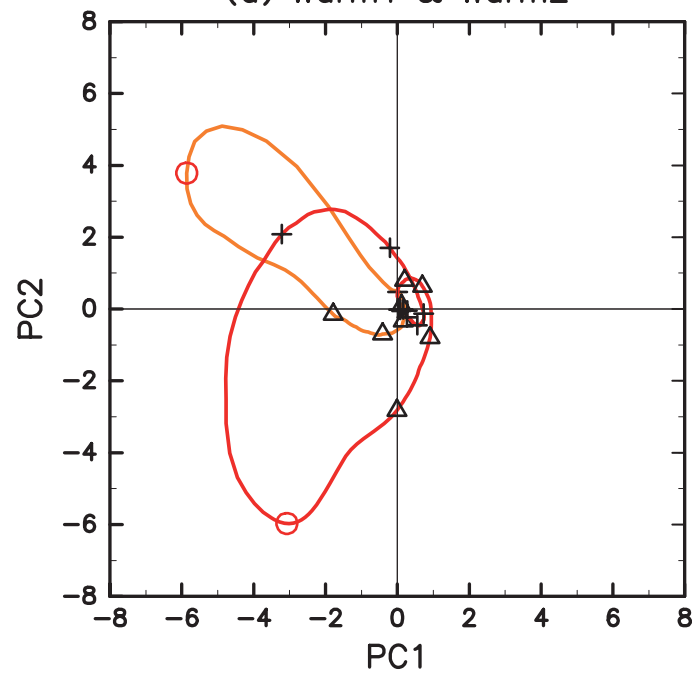

(b) Cold1 \& Cold2

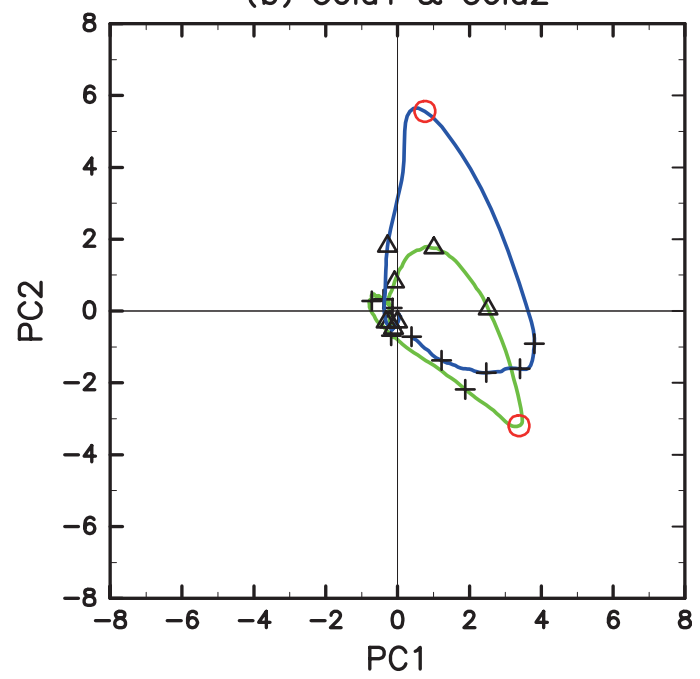

FIG. 4. (a) As in Fig. 1b, but for the trajectories of the composites of Warm1 (orange) and Warm2 (red) events shown in the bottom middle row of Figs. $3 a$ and b, respectively. Red circles indicate the key day (Lag $=0$ in Fig. 3) and a plus sign is marked every 20 days before the key day while a triangle is marked every 20 days after the key day. (b) As in (a), but for the regressed trajectories of the composites of Cold1 (green) and Cold2 (blue) events shown in the bottom middle row of Figs. 3c and 3d, respectively.

nonlinear nature of these oscillatory variations. Both trajectories of cold events also rotate anticlockwise, amplifying in the fourth quadrant. During the Cold1 event, the amplitude decreases quickly in the same fourth quadrant after attaining its maximum. During the Cold2 event, on the other hand, slow amplification continues over 80 days in the fourth quadrant, followed by comparatively fast phase variations for about 20 days until attaining the maximum amplitude. These composite trajectories show that each group of PJO events has its own oscillatory characteristics in the PJO space, and they do not correspond to the particular phase of any linear oscillator.

As for upward dynamical linkage in the slow variations, a positive peak of the AO index is found about a month or so prior to the key day of both Warm1 and Warm2 events, while a negative peak is found for both Cold 1 and Cold 2 events, as shown in the bottom panels in Figs. 3a-d. These relationships can be explained by correlative variations of planetary waves generated at the lower boundary in association with the AO (Taguchi and Yoden 2002; Nishizawa and Yoden 2004). For the period around the positive peak of the AO index, the larger-amplitude planetary waves give larger wave driving in the stratosphere to weaken the zonal-mean zonal wind in midlatitudes and increase adiabatic heating in the polar stratosphere (e.g., Haynes 2005). On the other hand, the situation is totally opposite for the period around the negative peak of the AO index. By composite analyses using reanalysis data, Kuroda (2008) showed that positive AO-like patterns appear before SSW while negative ones appear before VI. Our results are consistent with these relationships, even though the present model is an idealized mechanistic one as described in section 2 .

It is important to note the comparatively small but statistically significant negative peak of the AO index after the key day during the Warm 2 events for a discussion on the downward dynamical influence of PJO to the bottom of the troposphere. The highly significant positive AO index over a month before the key day cannot directly influence the negative index after the key day through solely tropospheric mechanisms because the time interval of these peaks of the AO index before and after the key day is much longer than the decorrelation time scale of about 30 days as stated in section 3c. Warm2 PJO events in the stratosphere are necessary to link the two peaks of the AO index through two-way interactions such as the troposphere-stratosphere-troposphere events demonstrated by Reichler et al. (2005). During Warm 1 events, on the other hand, a negative peak after the key day does not appear. This difference suggests the importance of the prolonged warm anomaly in the lower stratosphere during Warm2 events for the two-way interactions between the troposphere and the stratosphere.

\section{Conclusions}

Low-frequency variations in the winter stratosphere known as PJOs were investigated with a 14 000-yr-long 
dataset obtained with an idealized global mechanistic circulation model of the stratosphere and troposphere. After performing an EOF analysis of the northern polar temperature, 10647 PJO events were identified as largeamplitude, slow, and oscillatory (or rotating) variations in a two-dimensional phase space spanned by the leading two principal components as shown in Fig. 1. They were classified into four groups according to the phase of the state vector at each maximum (Fig. 2). Two groups are warm events (4970 Warm1 or 3488 Warm 2 events) with small or large phase variations (Fig. 4a). A composite analysis (Fig. 3) showed Warm2 events are similar to the typical PJO with slow downward propagation of a warm anomaly, which was originally found by Kuroda and Kodera (2001), while Warm1 events are characterized by the simultaneous occurrence of fast SSW events with a shorter time scale. With the same analysis procedure, two groups of cold events with opposite sign from the warm events were found in this study (820 Cold1 and 1369 Cold2 events). Both groups show slow downward propagation of a cold anomaly, with a longer time scale during Cold 2 events. The composite trajectories shown in Fig. 4 reveal that each group of PJO events has its own oscillatory characteristics and do not correspond to the particular phase of any linear oscillator.

Relationships of these PJO events to unfiltered stratospheric variations such as SSW and VI were investigated by making composites and histograms (Fig. 3). Most Warm1 events occur simultaneously with the first SSW during each winter, while Warm2 events occur about a month after the first SSW event. Similarly, more than half of the Cold1 events occur simultaneously with VI, while Cold 2 events occur about a month or two after a VI event and are followed by an SSW event. As for the relationship to slow tropospheric variations, statistically significant evidence of the downward dynamical influence of the PJO on the surface AO was obtained in the composites for Warm2 events.

This study is unique in that statistical analysis was done with enough significance by relying on a long dataset obtained by time-integrations of an idealized mechanistic circulation model. It is in a medium class in the hierarchy of numerical models between simple low-order models and complex general circulation models (e.g., Yoden et al. 2002), so that we can run the model with affordable computer resources. Some idealization of physical processes in the present model will help us to understand the essential dynamics of the low-frequency variations classified as four groups of PJO events-such a dynamical analysis remains to be studied in the near future. The concept of four groups of low-frequency PJOs and their analysis procedure developed in this study would be useful for further analysis of the real atmospheric data with limited length or similar datasets obtained with highend complex general circulation models.

Acknowledgments. This research was motivated by discussions with Peter Hitchcock of the University of Toronto when he stayed at our laboratory as a fellow of JSPS Summer Program in 2008. We are thankful for his comments on the original version of the manuscript. The figures were drawn by the software tools developed under the GFD Dennou Club Davis project.

\section{REFERENCES}

Baldwin, M. P., and T. J. Dunkerton, 1999: Propagation of the Arctic Oscillation from the stratosphere to the troposphere. J. Geophys. Res., 104, 30 937-30946.

Haynes, P., 2005: Stratospheric dynamics. Annu. Rev. Fluid Mech., 37, 263-293.

Kodera, K., Y. Kuroda, and S. Pawson, 2000: Stratospheric sudden warmings and slowly propagating zonal-mean zonal wind anomalies. J. Geophys. Res., 105, 12 351-12 359.

Kuroda, Y., 2008: Effect of stratospheric sudden warming and vortex intensification on the tropospheric climate. J. Geophys. Res., 113, D15110, doi:10.1029/2007JD009550.

_- , and K. Kodera, 1999: Role of planetary waves in the stratosphere-troposphere coupled valiability in the Northern Hemisphere winter. Geophys. Res. Lett., 26, 2375-2378.

_ and _ 2001: Variability of polar night jet in the Northern and Southern Hemispheres. J. Geophys. Res., 106, 20703 20723.

- and - 2004: Role of the polar night jet oscillation on the formation of the Arctic Oscillation in the Northern Hemisphere winter. J. Geophys. Res., 109, D11112, doi:10.1029/ 2003JD004123.

Limpasuvan, V., D. L. Hartmann, D. W. J. Thompson, K. Jeev, and Y. L. Yung, 2005: Stratosphere-troposphere evolution during polar vortex intensification. J. Geophys. Res., 110, D24101, doi:10.1029/2005JD006302.

McIntyre, M. E., 1982: How well do we understand the dynamics of stratospheric warmings? J. Meteor. Soc. Japan, 60, 37-65.

Nishizawa, S., and S. Yoden, 2004: A parameter sweep experiment on topographic effects on the annular variability. J. Meteor. Soc. Japan, 82, 879-893.

— , and -2005 : Distribution functions of a spurious trend in a finite length data set with natural variability: Statistical considerations and a numerical experiment with a global circulation model. J. Geophys. Res., 110, D12105, doi:10.1029/ 2004JD005714.

Reichler, T. P., P. J. Kushner, and L. M. Polvani, 2005: The coupled stratophere-troposphere response to impulsive forcing from the troposphere. J. Atmos. Sci., 62, 3337-3352.

Taguchi, M., and S. Yoden, 2002: Internal interannual variability of the troposphere-stratosphere coupled system in a simple global circulation model. Part I: Parameter sweep experiment. J. Atmos. Sci., 59, 3021-3036.

Thompson, D. W. J., and J. M. Wallace, 1998: The Arctic Oscillation signature in the winter geopotential height and temperature fields. Geophys. Res. Lett., 25, 1297-1300.

Yoden, S., M. Taguchi, and Y. Naito, 2002: Numerical studies on time variations of the troposphere-stratosphere coupled system. J. Meteor. Soc. Japan, 80, 811-830. 